\title{
Editorial
}

\section{System Simulation and Control in Engineering}

\author{
Her-Terng Yau, ${ }^{1}$ Maciej J. Ogorzalek, ${ }^{2}$ Frode Eika Sandnes, ${ }^{3}$ \\ Cheng Siong Lee (Vincent), ${ }^{4}$ and Yunhua $\mathrm{Li}^{5}$ \\ ${ }^{1}$ Department of Electrical Engineering, National Chin-Yi University of Technology, Taichung City, Taiwan \\ ${ }^{2}$ Department of Information Technologies, Jagiellonian University Krakow, Poland \\ ${ }^{3}$ Oslo and Akershus University, College of Applied Sciences, Oslo, Norway \\ ${ }^{4}$ Clayton School of Information Technology, Faculty of IT, Monash University, Australia \\ ${ }^{5}$ School of Automation Science and Electrical Engineering, BeiHang University, Beijing, China
}

Correspondence should be addressed to Her-Terng Yau; htyau@ncut.edu.tw

Received 2 September 2014; Accepted 2 September 2014; Published 22 December 2014

Copyright (c) 2014 Her-Terng Yau et al. This is an open access article distributed under the Creative Commons Attribution License, which permits unrestricted use, distribution, and reproduction in any medium, provided the original work is properly cited.

Technologies in the research field of system simulation and control have made great progress in recent decades, and system simulation and control has now become a popular term in the field of electrical/mechanic engineering. Many researchers in system simulation and control have made great efforts to develop methodologies for physical, engineering, and biological studies, and these research results have had great influence in the greater field of system simulation and control. With the advancement of computer hardware providing more powerful computing environments, system simulation and control researchers have been able to challenge solving larger and more complex problems. Driven by such motivation, the innovative methodologies of system simulation and control technologies are proposed not only in area of engineering but also in new paradigms in computer science. In addition, system simulation and control researchers have applied the developed methods to various real world problems such as robotic system. This special issue includes the mathematical and physical theories of the nonlinear dynamics analysis and control in physical, engineering, and biological studies and their various applications.

We strongly believe that the papers that are presented in this SI contribute greatly to the work of academic researchers, industry professionals, students, and anyone else who may be interested in this subject technology.

This special issue is composed of 61 nominated papers, carefully selected according to their subject and their merit. Fifty of the best papers to be presented at the IS3C 2014 Conference are included in the issue. This international conference is important to the development of new ideas and the promotion of research into advanced computer, consumer, and control technologies. Nearly 50 papers from around the world have been rigorously reviewed before being accepted for this special issue.

We thank all of the authors who contributed to this special issue.

Her-Terng Yau

Maciej J. Ogorzalek

Frode Eika Sandnes

Cheng Siong Lee (Vincent)

Yunhua Li 


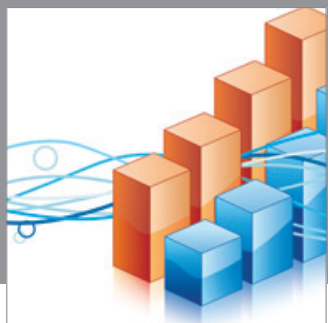

Advances in

Operations Research

mansans

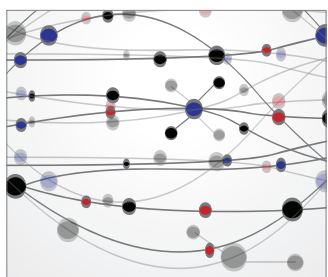

The Scientific World Journal
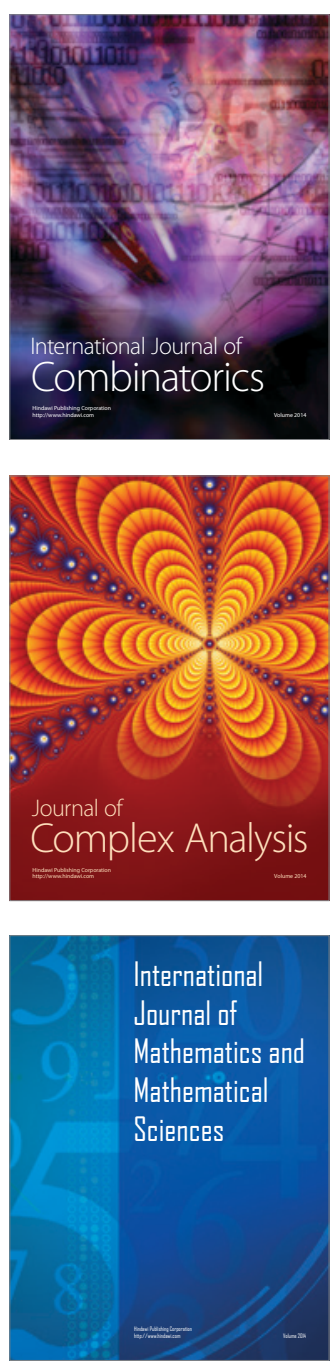
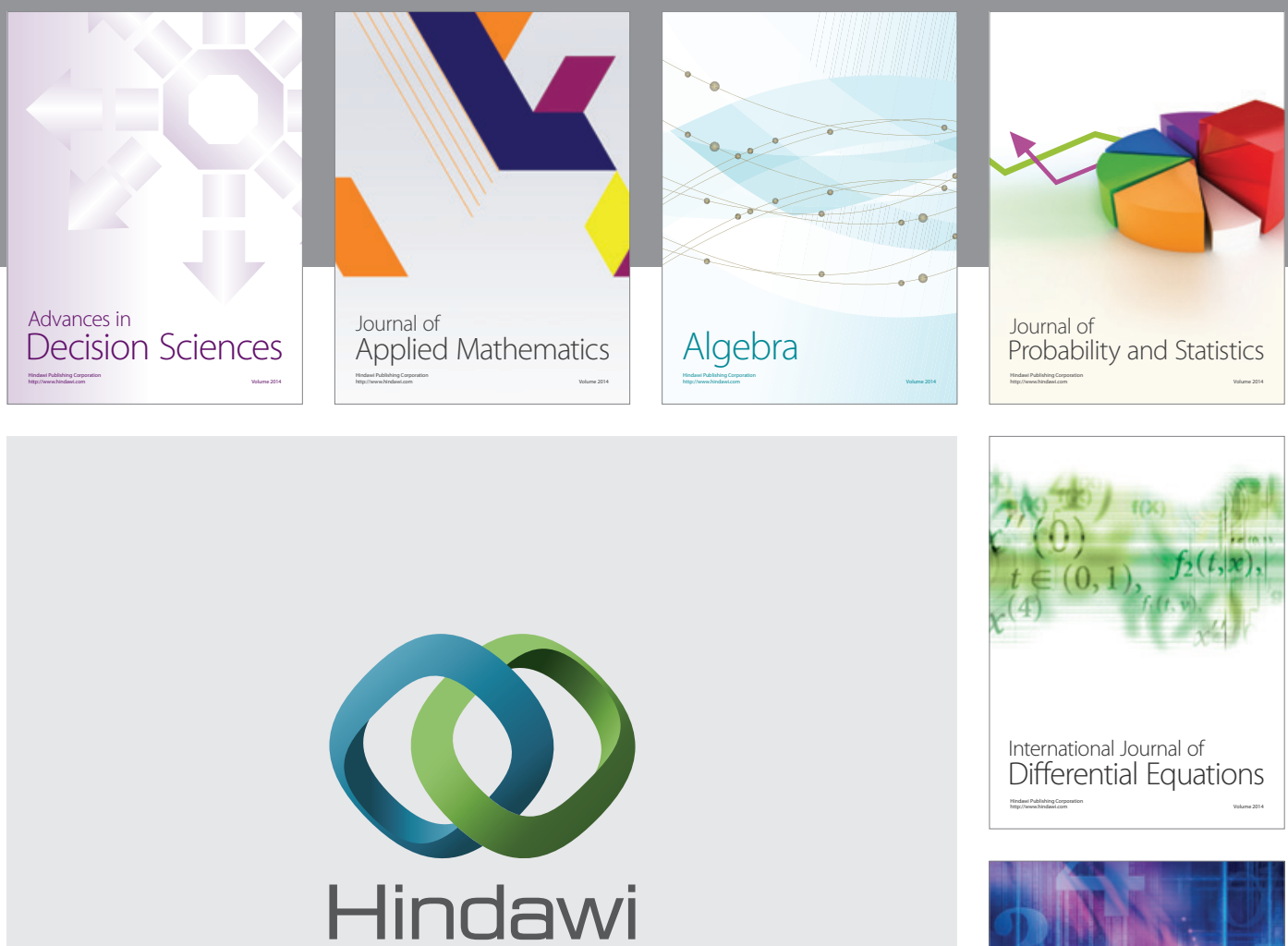

Submit your manuscripts at http://www.hindawi.com
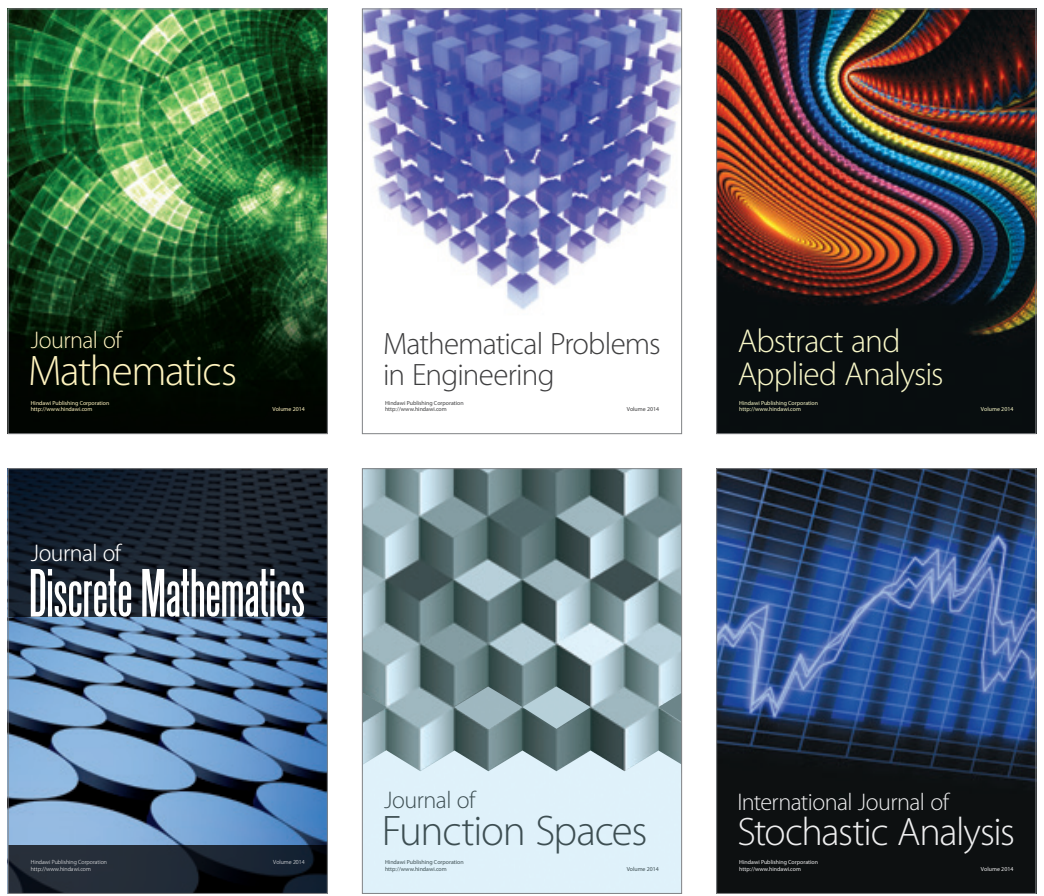

Journal of

Function Spaces

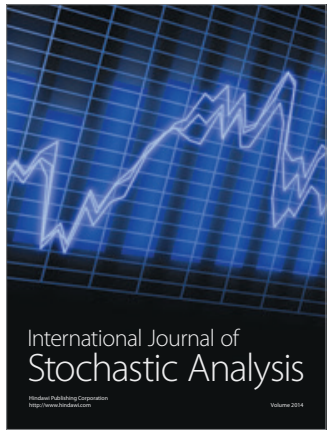

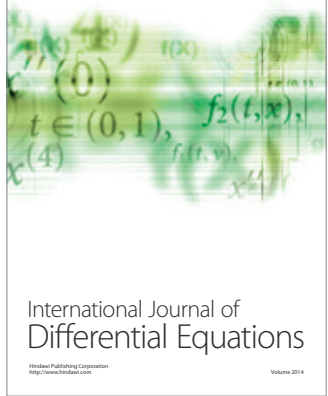
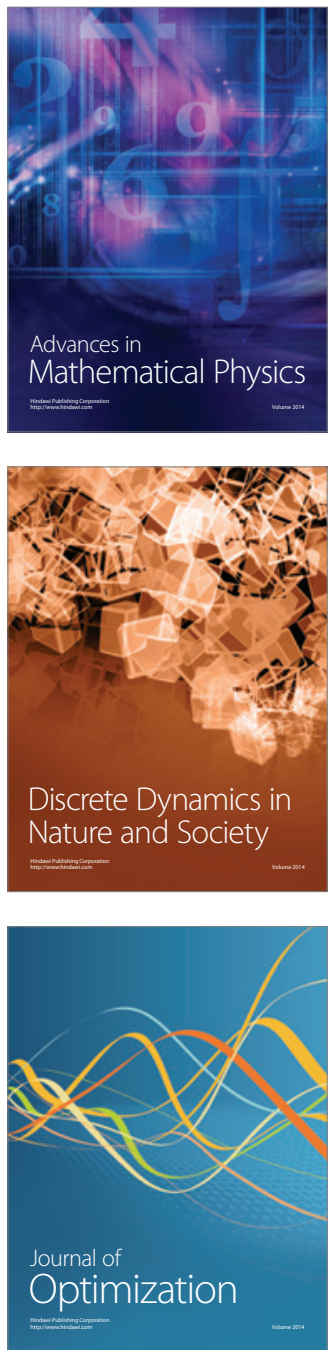